\title{
Empyema Thoracis: An Increasing Incidence with New Recognized Pathogen
}

\author{
Noor Sameh Darwich"1,* and Mark P. Anstadt ${ }^{2}$ \\ ${ }^{1}$ Miami valley Hospital, Dayton, Ohio, USA \\ ${ }^{2}$ Department of Surgery, Wright State University, Miami Valley Hospital, Dayton, Ohio, USA
}

${ }^{*}$ Corresponding author: Noor Sameh Darwich, Miami valley Hospital, One Wyoming Street Dayton, Ohio 45409, USA, E-mail: nsdarwich@gmail. com

Received: 02 Jan, 2020 | Accepted: 06 Feb, 2020 | Published: 11 Feb, 2020

Citation: Darwich NS, Anstadt MP (2020) Emphysema Thoracis: An Increasing Incidence with New Recognized Pathogen. J Infect Pulm Dis 5(1): dx.doi.org/10.16966/2470-3176.137

Copyright: (C 2020 Darwich NS, et al. This is an open-access article distributed under the terms of the Creative Commons Attribution License, which permits unrestricted use, distribution, and reproduction in any medium, provided the original author and source are credited.

\section{Abstract}

Pleural empyema is defined as purulent fluid formed in a pleural cavity, most commonly due to underlying pneumonia. Parapneumonic Effusions (PPE) include a spectrum ranging from uncomplicated, complicated effusion or empyema. Up to $30 \%$ of patients diagnosed with thoracic empyema have no evidence of pneumonia on CT imaging and no clear evidence of how the infected organism reached the pleural space. The most common bacteria isolated on the culture of empyema fluid are Streptococcus pneumonia, Staphylococcus aureus, anaerobes, and polymicrobial infection is not uncommon. However, Streptococcus intermedius/milleri is becoming more recognized as an important pathogen for empyema and lung abscess.

We describe a 54-year-old male with poor oral hygiene and significant history of alcohol and tobacco abuse who was presented to the emergency department with generalized malaise, cough, right-sided chest pain, loss of appetite, and unintentional weight loss for the previous several weeks. He was found to have a large right pleural effusion with compressive atelectasis on chest X-ray and CT imaging and underwent image-guided pleural catheter placement for drainage with the removal of $1100 \mathrm{ml}$ of malodorous, yellowish-greenish purulent fluid. Gram-positive cocci in chains were found on Gram stain of pleural fluid and aerobic culture of the fluid grew Streptococcus intermedius/milleri. The patient was started on antibiotics and eventually underwent Video-Assisted Thoracoscopy (VATS) which converted to Thoracotomy with total lung decortication. He recovered and was discharged home.

Keywords: Empyema; PPE; CAP; Streptococcus intermedius/milleri

\section{Introduction}

Recent epidemiological study indicated that the Incidence of empyema in the US had increased significantly from 1996 to 2008 $[1,2]$. The underlying reason for this increase was unknown. Empyema usually develops as a complication of underlying pneumonia in twothirds of empyema cases. Male to female ratio is 3:1. Up to $40-50 \%$ of patients with pneumonia develop some degree of pleural effusion, and $5-7 \%$ of community-acquired pneumonia patients will develop complicated parapneumonic effusion or empyema [3]. Communityacquired pneumonia is the most common preceding condition in both the elderly and children diagnosed with empyema. However, empyema can develop without the presence of pneumonia in cases such as penetrating or blunt trauma to the chest, the spread of nearby infection such as mediastinitis or subdiaphragmatic abscess, esophageal perforation, following Thoracic or abdominal surgery, or as a complication of Thoracostomy tube and thoracentesis $[4,5]$. Thoracostomy tube, for all indications, is considered a risk factor for pleural space infection and the risk is increased with an increase in the size of the tube and duration. However, this may be confounded by the fact that Thoracostomy tubes are commonly utilized as initial therapy for empyema. Notably, up to $5 \%$ of patients with Thoracostomy tube placement are felt to develop empyema due to iatrogenic pleural space infection. Primary thoracic empyema refers to pleural empyema in patients without clear evidence of underlying pneumonia or other recognized conditions to explain bacterial invasion to pleural space [6]. Streptococcus intermedius/milleri has been identified as a common pathogen for empyema and lung abscess [7]. The pathogenicity of Streptococcus intermedius/milleri causing empyema without pneumonia is still incompletely understood. Diagnostic thoracentesis and pleural fluid analysis in patients with pleural effusion can help detect complicated effusion early in the course, guide treatment with appropriate antibiotics and pleural space drainage which can prevent progression to empyema, avoid the need for more invasive procedures such as video-assisted thoracoscopy (VATS) or open thoracotomy with debridement and decortication [8]. It is important to note that adequate and complete drainage of empyema is central to effective therapy.

\section{Case Report}

A 54-year-old Caucasian male with a history of alcohol abuse and poor dentition was presented to the emergency department with a chief complaint of generalized malaise. The patient reported having symptoms of an upper respiratory tract infection approximately one 
month prior to his visit where he was very sick for two weeks. Since then, he lost his appetite and had 20 pounds' unintentional weight loss. He admits to night sweats, right-sided chest pain which subsided, and an occasional cough. On examination, his vital signs: BP 116/68 $\mathrm{mmHg}$, temp $98.9^{\circ} \mathrm{F}\left(37.2^{\circ} \mathrm{C}\right)$, pulse 116 per minute, respirations 20 per minute, and oxygen saturation $94 \%$ on room air, weight $77.1 \mathrm{~kg}$. He was Cachectic with poor dentition (Figure 1).

He denied any recent dental work or any chest trauma. Physical examination was noteworthy for decreased breath sounds and dullness to percussion throughout the right hemithorax. Laboratory data showed NA $129 \mathrm{mg} / \mathrm{dl}$, K $3.9 \mathrm{mg} / \mathrm{dl}$, BUN $5 \mathrm{mg} / \mathrm{dl}$, Creatinine $0.5 \mathrm{mg} / \mathrm{dl}$ (normal reference range $0.6-1.30 \mathrm{mg} / \mathrm{dl}$ ), Glucose $112 \mathrm{mg} /$ dl. WBC 30.3 (normal reference range 4.0-10.5 103/UL, Hemoglobin $13.5 \mathrm{~g} / \mathrm{dl}$ (normal reference range 12.0-15.6 g/dl) with normal liver function tests. Chest-X ray showed large right pleural effusion (Figure 2 ) and CT chest confirmed large loculated right pleural effusion and compressive atelectasis (Figure 3).

The patient was started on intravenous antibiotics, piperacillin/ tazobactam. Further, he underwent CT guided pleural small caliber catheter placement of right pleural cavity with the removal of $1100 \mathrm{ml}$ of malodorous, yellowish/greenish purulent fluid (Figure 4).

Pleural fluid analysis demonstrated $\mathrm{PH} 6, \mathrm{LDH}>4000 \mathrm{U} / \mathrm{L}$, glucose $72 \mathrm{mg} / \mathrm{dl}$, total protein $2.9 \mathrm{mg} / \mathrm{dl}$, amylase 14, WBC $603.889 \mathrm{~K} / \mathrm{MM} 3$ with $99 \%$ granulocytes and $1 \%$ lymphocyte. Gram-positive cocci were found on Gram stain of pleural fluid, and aerobic culture of the fluid grew Streptococcus intermedius while anaerobic culture of pleural fluid remained negative, and blood culture was negative. Pleural fluid cytology demonstrated numerous degenerating neutrophils with an extra and intracellular bacterial organism and was negative for malignancy. The antibiotic was changed to ceftriaxone, but a repeat in Chest-X ray showed persistent loculated right pleural effusion. He underwent right video-assisted thoracoscopy (VATS) under general anesthesia using a double-lumen endotracheal tube for isolation of his lung. On entry into the right chest cavity, there was gross pus which was evacuated. Inspection with the scope revealed a clear thick pulmonary rind covering the lung that could not be removed in a purely thoracoscopic fashion. Therefore, VATS was converted to a muscle sparing posterior lateral thoracotomy with total lung decortication and washout of pleural space for stage III right empyema thoracis with entrapped lung. Pleural surgical specimens showed necrosis and granulation tissue with acute inflammation (Figure 5) as well as fibrotic changes (Figure 6).

The patient recovered well after surgery but with persistent air leak while the chest tube on the water seal. Due to persistent air leak for several days, the patient underwent redo VATS on post-op day 7 with washout of pleural space and placement of $28 \mathrm{~F}$ chest tube connected to suction at $-40 \mathrm{CM} \mathrm{H}_{2} \mathrm{O}$. The suction was reduced gradually by 5 $\mathrm{CM} \mathrm{H}_{2} \mathrm{O}$ and eventually to the water seal and the Pleura-vac changed to mini-500 device to maintain the water seal. Follow up Chest-X ray (Figure 7) showed no pneumothorax and the patient was discharged home with right chest tube to water seal connected to mini portable drainage collection unit. His antibiotics were eventually changed to oral amoxicillin-clavulanic acid to complete a total of 4 weeks. He was advised to improve his oral dentition and follow up with a dentist. His chest tube was eventually removed 4 weeks after discharge and a follow up Chest-X ray (Figure 8) showed significant improvement with minimal residual pleural thickening/scaring at the right base.

\section{Discussion}

Primary pleural empyema without identifying an underlying cause can account for up to $30 \%$ of patients with empyema [9]. There are uncertainties about the mechanism of primary pleural empyema in the absence of underlying pneumonia, recent surgery, or trauma. Clinically overt pneumonia or indolent lung abscess, which can drain spontaneously to the pleural space infecting the pleural cavity while the abscess itself can heal after draining is a potential process that can explain the transmission of the bacteria into the pleural space. Another possibility is translocation of the bacteria from the airways, after oral aspiration, to the pleural space without causing lung infection or pneumonia can theoretically occur through parenchymal lung tissue and visceral mesothelial cells of the pleura [10]. Finally, hematogenous seeding of bacteria to pleural space, which can occur as a result of transient bacteremia from dental plaque and periodontal tissue or gingivitis even after simple dental manipulation (toothbrush) can lead to primary pleural empyema without causing endocarditis $[11,12]$. Transient bacteremia may occur in $80 \%$ of patients with poor oral hygiene and periodontal disease even after aggressive toothbrush use and may produce abscess formation in sites other than the heart valve [13].

Gram stain and culture of pleural fluid is positive in only $50-60 \%$ of empyema cases with the rest being culture negative and this may be due to prior use of antibiotics or anaerobic pathogens that require special anaerobic culture media to grow on. Streptococcus pneumonia, Staphylococcus aureus, and anaerobes were the most common causative isolates in empyema patient in past studies. However, recent data indicate that Streptococcus milleri is an emerging pathogen and the most common isolate found recently in adults with empyema accounting for $30-50 \%$ of cases in UK, Canada, New Zealand and the U.S. Streptococcus milleri is part of the streptococcal group which includes Streptococcus anginosus, Streptococcus costallatus, and Streptococcus intermedius. It is gram-positive catalase negative facultative anaerobe and part of the normal flora of the mouth, gastrointestinal tract, skin, genitourinary system and rarely isolated as a pathogen in community-acquired pneumonia. The virulent mechanism of $S$. milleri has not been fully determined [14]. It is typically seen as a part of polymicrobial pneumonia and has a tendency to cause lung abscess and empyema. Empyema due to Mycobacterium tuberculosis is seen more in developing countries where there is a higher risk for Mycobacterial infection. Other responsible agents for empyema are Actinomycetes, Mucormycosis, and Nocardia which are reported mostly in immunocompromised patients. Fungal empyema, which includes Histoplasmosis and Coccidiomycosis, is seen mostly in patients with severe immunodeficiency. Parasitic causes of empyema include amoebic liver abscess ruptured through the diaphragm to the pleural cavity [15]. Risk factors for developing empyema include alcohol abuse, diabetes mellitus, neoplasm, poor dentition, cerebrovascular accident, swallowing dysfunction with aspiration, underlying pulmonary disease such bronchiectasis, chronic obstructive pulmonary disease, post-obstructive pneumonia from underlying endobronchial lesion such as malignancy versus foreign body aspiration and male gender [16-18]. Poor host immunity or a delay in diagnosis and treatment of pneumonia might be other reasons for developing empyema. Interestingly, the use of inhaled steroids in patients with COPD or asthma found to decrease the incidence of developing parapneumonic effusion [19]. Presenting symptoms of empyema are non-specific and depend on the time of presentation after primary infection of the pleural cavity which can be acute, subacute, or chronic including chest pain, cough, shortness of breath, fever, night sweats, as well as weight loss and malaise [20].

The American Thoracic Society has staged empyema into 3 stages: stage I: exudative effusion without loculation (pre-empyema) usually 


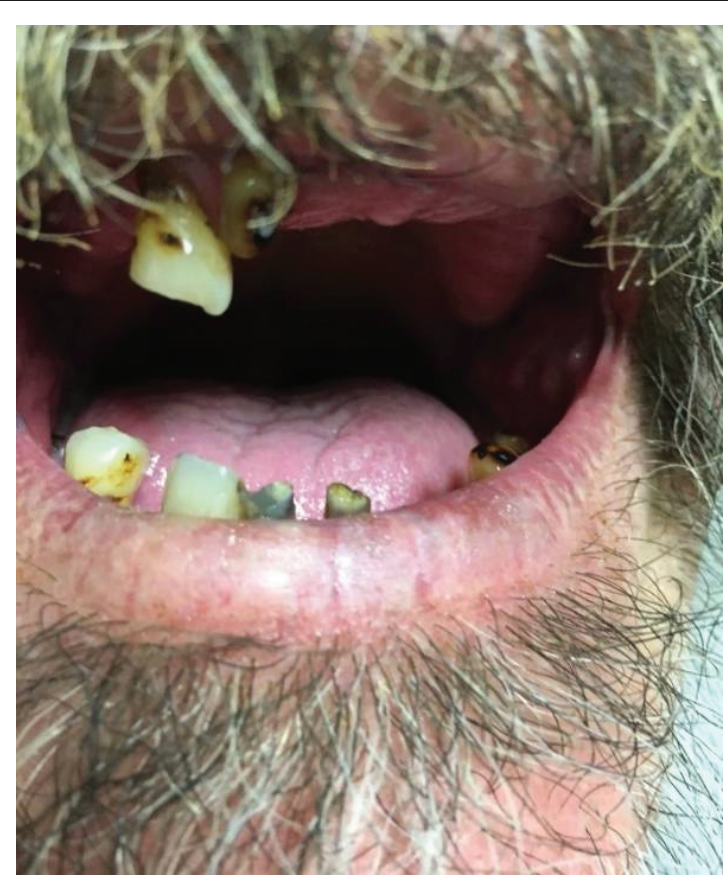

Figure 1: Poor dentition.

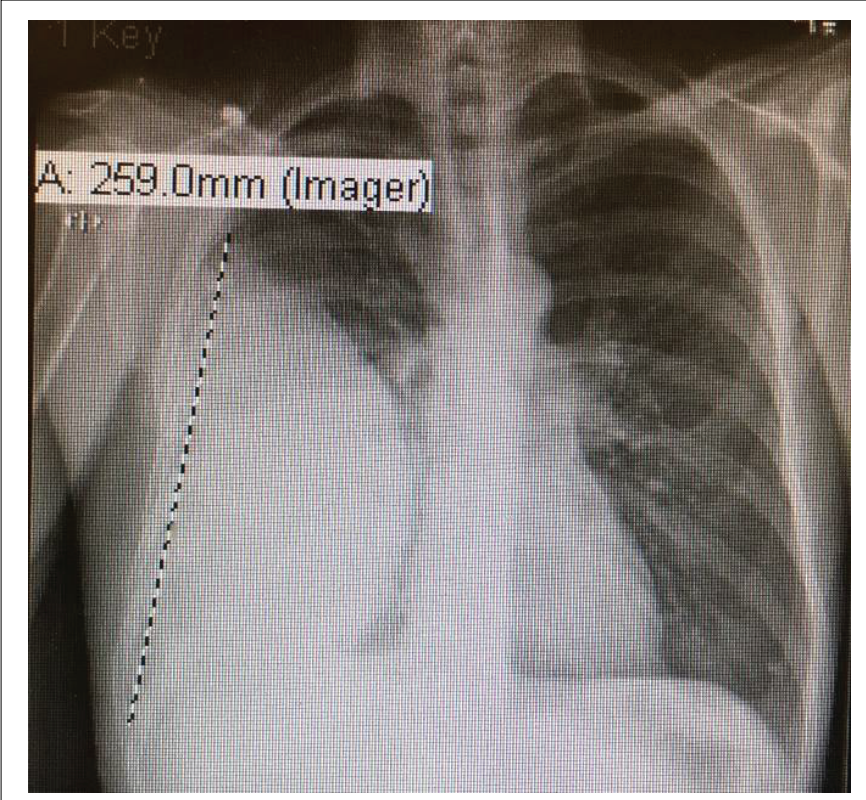

Figure 2: Chest-X ray with large right pleural effusion

sterile fluid, represent the uncomplicated PPE, last for 24-72 hours before it progresses to stage II: fibrinopurulent phase with turbid or frank purulent pus with formation of fibrin clots and membrane accompanied by bacterial invasion and loculations within pleural space can be seen. This stage lasts for 7-10 days then progresses to stage III: organizing pleural fibrosis with entrapped lung as the thick pleural peel is formed causing Fibrothorax. This stage occurs within 2-4 weeks after the initial process. Although this classification explains fully the underlying pathophysiology of empyema progression, it is not based on clinical ground for primary care physicians to use as

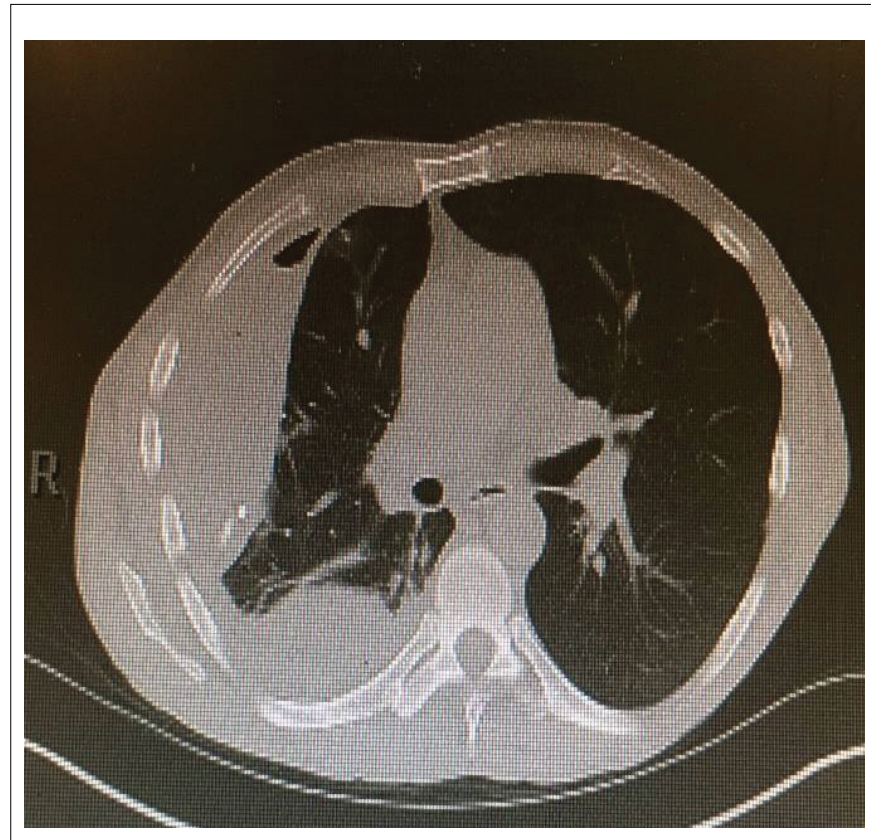

Figure 3: CT- chest showed large loculated right pleural effusion and compressive atelectasis.

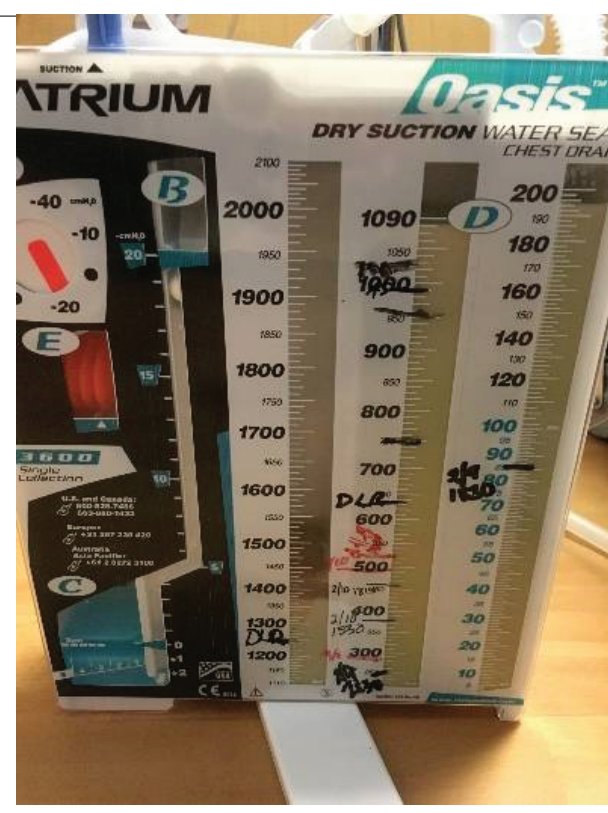

Figure 4: Yellowish/greenish purulent pleural fluid.

guidance to when they should refer their patient with parapneumonic effusion to thoracic surgery team [21].

Treatment depends on the stage, and clinical condition of the patient and includes initial intravenous antibiotics for all stages which should be broad spectrum at the start of therapy covering Gram positive, Gram-negative and anaerobes, then narrow spectrum once the result of cultures and sensitivities are available. If pleural fluid is culture negative, then the antibiotic coverage depends on clinical presentation to cover both aerobic and anaerobic pathogens. Antibiotic treatment is usually a prolonged course up to 4 weeks based 


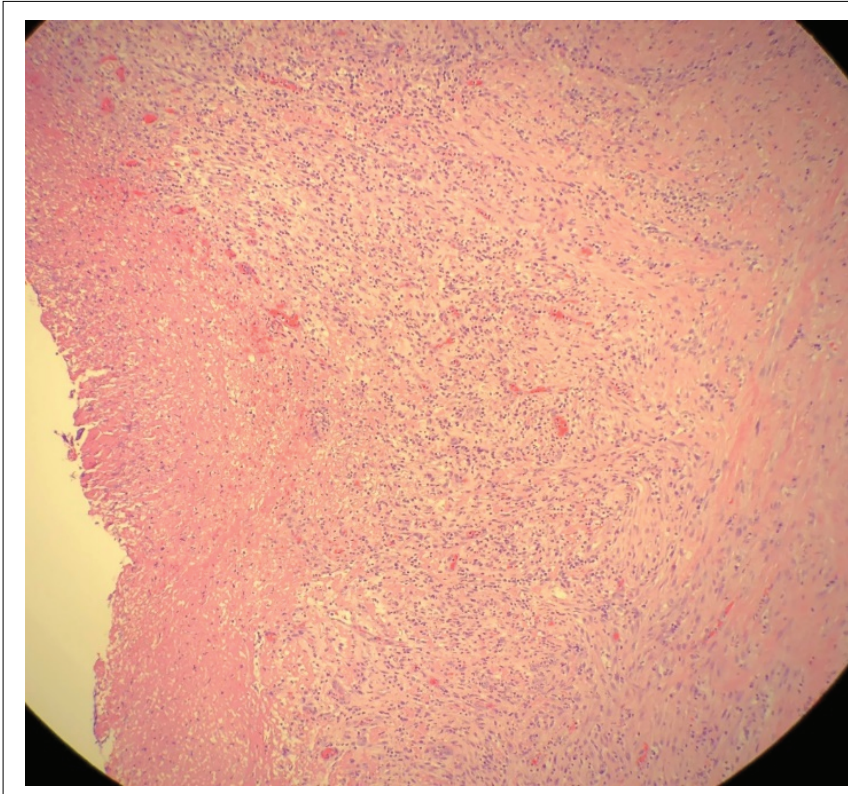

Figure 5: Pleural surgical specimen with necrosis and granulation tissue with acute inflammation.

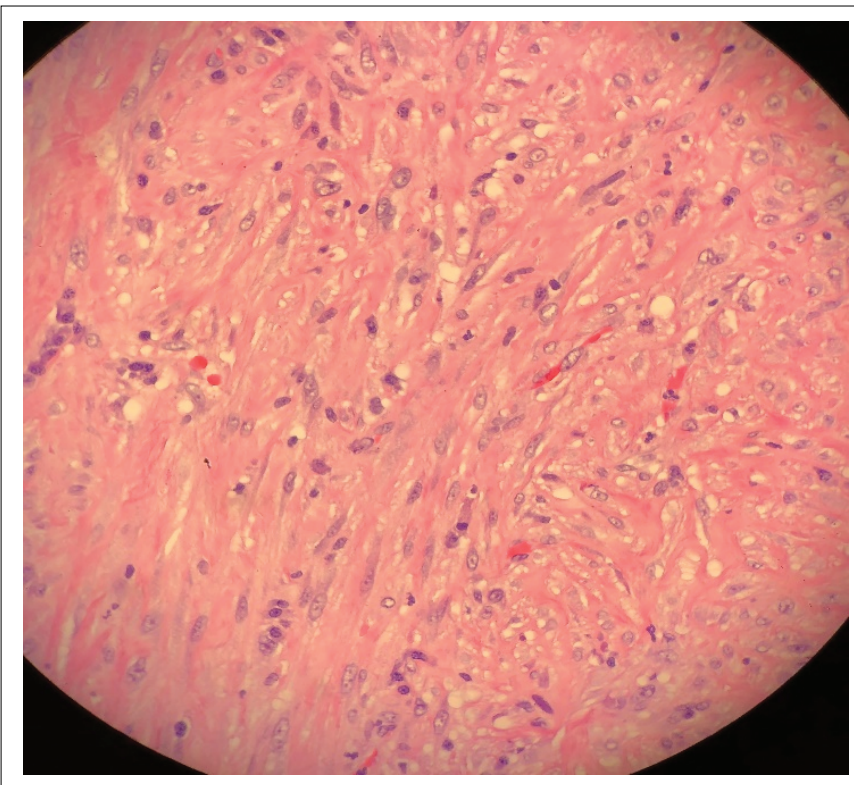

Figure 6: Pleural surgical specimen with fibrotic changes.

on clinical and radiological improvement [22]. Pleural drainage for stage I and stage II empyema could be achieved with small or mediumsized pleural catheter placement under imaging guidance ultrasound or CT, which is as efficacious as large tube thoracostomy in early stages of complicated pleural effusion. Drainage needs to be done as soon as possible when the fluid becomes complicated [23]. The use of local thrombolytic (fibrinolytic) TPA \pm Mucolytic (DNAse) has been used for stage II empyema with loculated effusion which helps dissolve pleural adhesions and break down septation and fibrin deposition on pleura to decrease the viscosity of the pleural fluid [24]. Combination of TPA (tissue plasminogen activated Alteplase) and (recombinant human deoxyribonuclease) rhDNAse are more effective than either

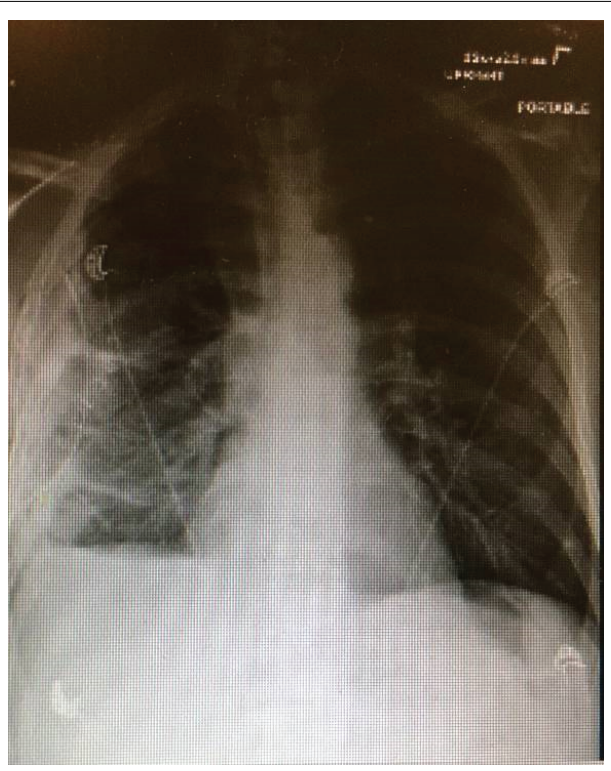

Figure 7: Chest-X ray post redo VATS with right sided chest tube, right pleural thickening/small pleural effusion/atelectasis but no pneumothorax.

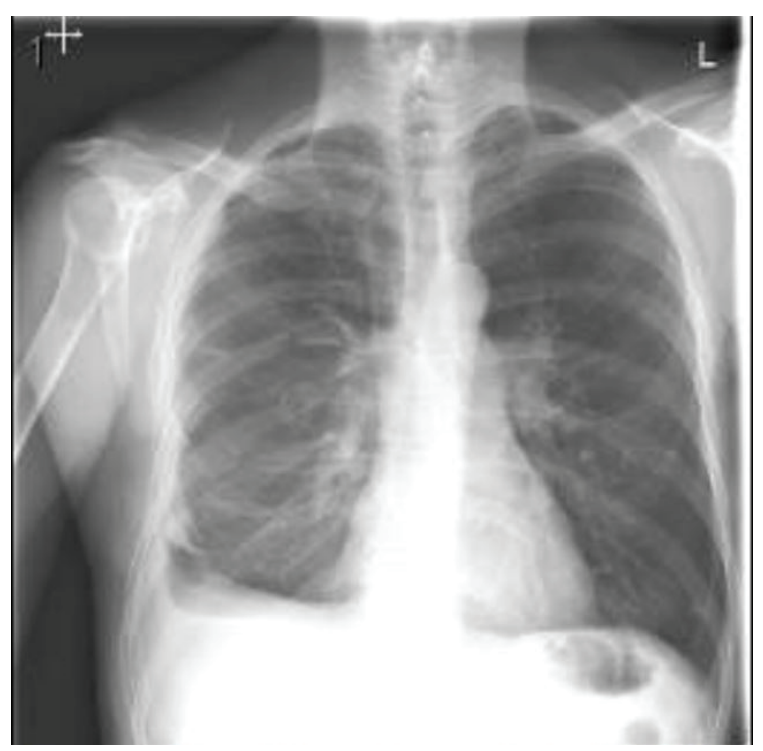

Figure 8: Chest- $X$ ray after discharge with removal of chest tube, showed significant improvement with minimal residual pleural thickening/scaring at right base of the lung.

agent alone in several studies by improving fluid drainage and decreasing the need for surgery in some cases $[25,26]$. A recent study found the presence of pleural thickening and abscess or necrotizing pneumonia predict failure in the administration of intrapleural tPA/ DNase in patients with complicated parapneumonic effusion or empyema suggesting the need for early surgical intervention to reduce mortality, hospital stay, and costs [27]. VATS with debridement and or decortication is recommended in patient with Stage II empyema and should be done early when pleural fluid drainage fails to expand the lungs. Many experts have recommended VATS as a first surgical approach for all patients with stage II empyema not only for drainage and debridement but also for decortication before empyema progress 
to stage III making decortication difficult to achieve with VATS and demanding conversion to open thoracotomy with decortication as in our case [28]. Early VATS has been associated with better outcome with quick recovery, short hospital stay, less air leak, less pain postoperatively, and lower mortality rate compare to open thoracotomy $[29,30]$. The American Association for thoracic surgery consensus guidelines for the management of empyema published in 2017 recommended (Class IIa-LOE B) Video-assisted thoracoscopic surgery (VATS) as a firstline approach in all patients with stage II acute empyema [31]. Timing of surgery is critical, as delayed surgical referral may mandate open thoracotomy as well as increases the chance of VATS conversion to open thoracotomy $[32,33]$. Open thoracotomy with decortication is reserved for complex cases with chronic empyema. Mortality in empyema varies and remains high, up to $10-20 \%$ depending on many factors such as host defense immunity, pathogens, early drainage, and early use of antibiotics.

\section{Conclusion}

It has been reported that the incidence of empyema was increased between 1996 to 2008 in both adults and children, compared to prior years, and the main reason remains to be determined [34,35]. It is important for primary care physicians to be aware of this possible complication of pneumonia in order to closely monitor patients treated for pneumonia in the outpatient and inpatient settings. This should be done with follow up Chest-X ray to identify those who need diagnostic aspiration of pleural fluid which is recommended if the effusion is moderate in size, if there is poor clinical improvement with the use of antibiotics, or if there is a high suspicion of complicated effusion or empyema in high-risk patient groups such as alcoholics and patients with swallowing dysfunction [36]. Awareness of possible primary thoracic empyema without underlying pneumonia in a patient presenting with nonspecific symptoms and only pleural effusion on imaging study needs to be emphasized in order to avoid delay in surgical referral. Early adequate pleural drainage and antibiotic use is the key to a better outcome. Normally VATS can serves as an early minimally invasive means to accomplish not only as effective drainage but also for debridement and decortication. Otherwise, Thoracotomy with formal decortication is eventually required in patients for whom antibiotic and less invasive means of pleural drainage fail to expand the lung $[37,38]$. We support the notion that early VATS is the preferred intervention in most patients with stage II empyema; however, more studies are needed to confirm this recommendation. The only exception may be in the pediatric Empyema thoracis when conservative treatment with effective antibiotics alone or with pleural drainage may be sufficient without the need for VATS or open thoracotomy with good long term outcomes [39].

\section{Acknowledgment}

The authors thank Thomas E. Herchline MD for reviewing the manuscript and for his valuable constructive suggestions and support.

\section{References}

1. Grijalva CG, Zhu Y, Nuorti JP, Griffin MR (2011) Emergence of parapneumonic empyema in the USA. Thorax 66: 663-668.

2. Finley C, Clifton J, Fitzgerald JM, Yee J (2008) Empyema: an increasing concern in Canada. Can Respir J 15: 85-89.

3. Ahmed RA, Marrie TJ, Huang JQ (2006) Thoracic empyema in patients with community-acquired pneumonia. Am J Med 119: 877883.

4. Taryle DA, Potts DE, Sahn SA (1978) The incidence and clinical correlated of parapneumonic effusions in Pneumococcal pneumonia. Chest 74: 170-173.
5. Brims FJ, Lansley SM, Waterer GW, Lee YC (2010) Empyema Thoracis: new insights into an old disease. Eur Respir Rev 19: 220-228.

6. Mandal AK, Thadepalli H, Mandal AK, Chettipally U (1998) Outcome of primary empyema thoracis: theraputic and microbiology aspects. Ann Thorac Surg 66: 1782-1786.

7. Shinzato T, Saito A (1995) The Streptococcus milleri group as a cause of Pulmonary infections. Clin Infect Dis 21: S238-S243.

8. Sahn SA (2007) Diagnosis and management of Parapneumonic effusions and empyema. Clin infect Dis 45: 1480-1486.

9. Lee YCG, Fitzgerald DB (2018) Pleural biopsy to capture causative microbe: A new piece of the Pleural Infection Jigsaw. Chest 154: 743-745.

10. Corcoran JP, Wrightson JM, Belcher E, De Camp MM, Feller-Kapman $D$, et al. (2015) Pleural infection: past, present, and future directions. Lancet Respir Med 3: 563-577.

11. Roy WJ Jr, Roy TM, Davis GJ (1991) Thoracic empyema due to Streptococcus intermedius. J Ky Med Assoc 89: 558-562.

12. Petersdorf RG (1978) Antimicrobial prophylaxis of bacterial endocarditis. Prudent caution or bacterial overkill? Am J Med 65: 220-223.

13. Iskandar SB, Al Hasan MA, Roy TM, Byrd RP Jr (2006) Streptococcus intermedius: An unusual cause of a primary Empyema. Tenn Med 99: 37-39.

14. Wong CA, Donal F, Macfarlane JT (1995) Streptococcus milleri Pulmonary disease: a review and clinical description of 25 patients. Thorax 50: 1093-1096.

15. Falguera M, Carratala J, Bielsa S, Garcia-vidal C, Ruiz-Gonzalez A, et al. (2011) Predictors factors, microbiology and outcome of patient with parapneumonic effusion. Eur respir J 38: 1173-1179.

16. Lu HY, Liao KM (2018) Risk of empyema in patients with COPD. Int J Chron Obstruct Pulmon Dis 13: 317-324.

17. Chalmers JD, Singanayagam A, Murray MP, Scally C, Fawazi A, et al. (2009) Risk factors for complicated parapneumonic effusion and empyema on presentation to hospital with community-acquired pneumonia. Thorax 64: 592-597.

18. Shen TC, Lin CY, Lin CL, Chen CH, Tu CY, et al. (2017) Risk of developing pleural empyema in patients with stroke: a propensity-matched cohort study. Intern Emerg Med 12: 1131-1138.

19. Sellares J, Lopez-Giraldo A, Lucena C, Cilloniz C, Amaro R, et al. (2013) Influence of previous use of inhaled corticoids on the development of pleural effusion in community-acquired pneumonia. Am J Respir Crit Care Med 187: 1241-1248.

20. Briant RE, Salmon CJ (1996) Pleural empyema. State of the art clinical article. Clin Infect Dis 22: 747-764.

21. Anstadt MP, Guill CK, Ferguson ER, Gordon HS, Soltero ER, et al. (2003) Surgical versus Nonsurgical Treatment of Empyema Thoracis: An Outcomes Analysis. Am J Med Sci 326: 9-14.

22. Hamm H, Light RW (1997) Parapneumonic effusion and empyema. Eur Respir J 10: 1150-1156.

23. Girdhar A, Shujaat A, Bajwa A (2012) Management of Infectious processes of the Pleural Space: A Review. Pulm Med 2012: 816502.

24. Zhu Z, Hawthorne ML, Guo Y, Drake W, Bilaceroglu S, et al. (2006) Tissue plasminogen activator combined with human recombinant deoxyribonuclease is effective therapy for empyema in a rabbit model. Chest 129: 1577-1583. 
25. Simpson G, Rooms D, Heron M (2000) Effect of streptokinase and deoxyribonuclease on viscosity of human surgical and empyema puss. Chest 117: 1728-1733.

26. Rahman NM, Maskell NA, West A, Teoh R, Arnold A, et al. (2011) Intrapleural use of tissue plasminogen activator and DNase in pleural infection. N Eng J Med 365: 518-526.

27. Khemasuwan D, Sorensen J, Griffin DC (2018) Predictive variables for failure in administration of intrapleural tissue plasminogen activator/ deoxyribonuclease in patient with complicated parapneumonic effusion/Empyema. Chest 154: 550-556.

28. Shen KR (2018) Surgical management of Pleural empyema. Shanghai Chest 2: 29.

29. Chambers A, Routledge T, Dunning J, Scarci M (2010) Is video-assisted thoracoscopic surgical decortication superior to open surgery in the management of adults with primary empyema? Interact Cardiovasc Thorac Surg 11: 171-177.

30. Marks DJB, Fisk MD, Koo CY, Pavlou M, Peck L, et al. (2012) Thoracic empyema: a 12-year study from UK tertiary Cardiothoracic Referral center. PLos One 7: e30074.

31. Shen KR, Bribriesco A, Crabtree T, Denlinger C, Eby J, et al. (2017) The American Association for Thoracic Surgery consensus guidelines for the management of empyema. J Thorac Cardiovasc Surg 153: e129-e146.
32. Tong BC, Hanna J, Toloza EM, Onaitis MW, D’Amico TA, et al. (2010) Outcomes of video-assisted thoracoscopic decortication. Ann Thorac Surg 89: 220-225.

33. Reichert M, Hecker M, Witte B, Bodner J, Padberg W, et al. (2017) Stage-directed therapy of Pleural empyema. Langenbecks Arch Surg 402: 15-26.

34. Burgos J, Falco V, Pahissa A (2013) The increasing incidence of empyema. Curr Opin Pulm Med 19: 350-356.

35. Grijalva CG, Nourti JP, Zhu Y, Griffin MR (2010) Increasing incidence of empyema complicating childhood community-acquired pneumonia in the United States. Clin Infect Dis 50: 805-813.

36. Lardinois D, Gock M, Pezzata E, Buchli C, Rousson V, et al. (2005) Delayed referral and gram-negative organisms increase the conversion thoracotomy rate in patient undergoing video-assisted thoracoscopic surgery for empyema. Ann Thorac Surg 79: 18511856.

37. Davies CW, Kearney SE, Glesson FV, Davies RJ (1999) Predictors of outcome and long-term survival in patients with pleural infection. Am J Respir Crit Care Med 160: 1682-1687.

38. Light RW (2006) Parapneumonic effusions and empyema. Proc Am Thorac Soc 3: 75-80.

39. Cohen E, Mahant S, Dell SD, Traubici J, Ragone A, et al. (2012) The Long-term Outcomes of Pediatric Pleural Empyema : a prospective study. Arch Pediatr Adolesc Med 166: 999-1004. 